\title{
Analytical Study of Nonlinear Vibrations of the Tympanic Membrane by Homotopy Perturbation Method
}

\author{
Masoud Rahmani ${ }^{1 *}$, Amin Moslemi Petrudi ${ }^{1}$, Ionut Cristian Scurtu ${ }^{2}$ \\ ${ }^{1}$ Department of Mechanical Engineering, Tehran University, Iran \\ ${ }^{2}$ Mircea cel Batran Naval Academy, Constanta, Romania \\ Email: Masoud.rahmani71@gmail.com
}

\begin{abstract}
Nonlinear vibration problems are generally of great importance in physics, mechanical structures, and other engineering research. This type of equation is very difficult and time-consuming. Researchers, therefore, focus on analytical and numerical methods; In this paper, using the new Newton-Harmonic Balance analytical method, which includes a combination of Newton methods and the harmonic balance method, the common Homotopy Perturbation method is used to solve the nonlinear equation of human tympanic membrane vibration and are compared and validated.
\end{abstract}

Keywords: Homotopy perturbation, Human tympanic membrane, Nonlinear vibrations, Harmonic balance.

\section{Introduction}

Nonlinear vibration problems are more and more used in solving difficult problems. In this article we use the Newton Harmonic Balance Method (NHBM), which is a combination of the Newton method and harmonic balance method to solve the problem of nonlinear vibrations of the human tympanic membrane. Among the researches that have been done so far in this field, we can mention the work of $\mathrm{Hu}$, which has provided a real solution for the second-order nonlinear equation [1]. The approximate solution of the periods of the Helmholtz-Duffing equation, which is also used for the tympanic membrane, has been investigated by Askari et al. [2]. Ji-Huan He first introduced the perturbation homotopy method in 1998 [3] and is used for a series of ordinary nonlinear differential equations (ODE), including the third-order nonlinear order Duffing equation, and it was shown that this new method It has an advantage over the classical perturbation method and the initial guess can be freely approximated by unknown constants. These constant values can be determined by many methods and also the approximations obtained from this method are not valid only for small values of the perturbation parameter but also for large values. In 2000, he used the homotopy perturbation method for a series of nonlinear vibrational equations, including equations with different damping factors, as well as an example of a singularity equation, and listed the advantages of this method over the classical perturbation method, then in 2004. Used this method for quantitative analysis of discontinuous nonlinear oscillators [4]. In 2005, with the help of his colleagues, he used this method to analyze a new nonlinear dynamic model of a spinning machine [5] and in his research, he obtained the optimal angle of convergence of two threads in the equilibrium position of $90^{\circ}$ and resonance in $270^{\circ}$. In 2005, L. Cveticanin's homotopy method was used to solve the ODE differential equations of complex numbers with nonlinear order of the cubic equation type [6]. So that the initial nonlinear ODE differential equation became a system of linear equations and the results were in good agreement with the exact analytical results. In 2006, he used this method to solve the nonlinear ODE differential equations of the quadratic and cubic nonlinear order equations of nonlinear oscillators. The initial conjecture was considered as a Jacobi elliptic function and the result was 
compared with a numerical solution [7] and it was observed that the initial conjecture of the algorithm is very simple and has very high accuracy. In 2006, Turgut Ozis et al. Used the homotopy perturbation method for the strong nonlinear van der pol oscillator [8]. In 2006, Shu-Li Me et al combined nonlinear dynamic dynamical systems with a degree of freedom by combining the homotopy perturbation method and the precise integrating method (PIM). And they concluded that the new method was superior to the first two methods. In 2006, A. Belendez et al used the homotopy method to analyze conservative nonlinear oscillators [9]. Other researches in the field of perturbation homotopy method include wave cases, heat transfer, boundary value problems in physics-mechanics, and dynamic models of growth phenomena in biology, Laplace transform, integral equations. Also, several modifications have been obtained by researchers for the homotopy perturbation method. A comparison between the homotopy perturbation method and Homotopy Analyzes Method (HAM) has also been done by Shijun Liao [10]. In general, because in most of the abovementioned references, researchers have done a little analysis of the problem and obtained the analytical answer of the equations, the result of their research is only the advantages of the homotopy method of perturbation and that this analytical method can solve most nonlinear science equations. They are engineers. It is important to note that having an analytical answer to nonlinear problems helps to understand the physics of the problem and makes this result valuable.

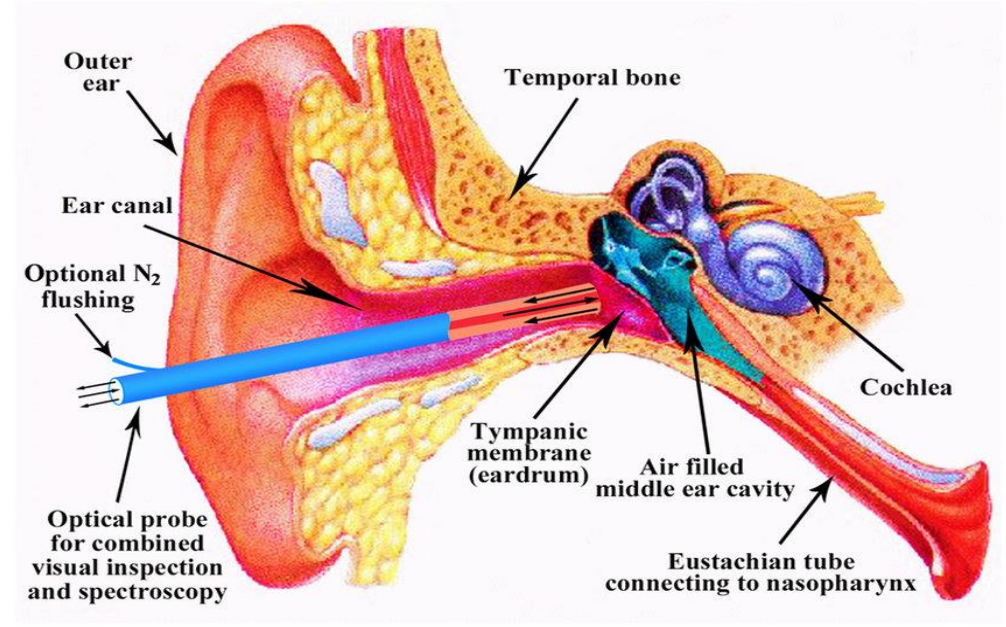

Fig.1. Ear structure due to vibration [10].

\section{Basis of the homotopy perturbation method}

To show the principles of this method, the following nonlinear differential equation:

$$
\mathrm{A}(\mathrm{u})-\mathrm{f}(\mathrm{r})=0, \quad \mathrm{r} \in \Omega,
$$

With boundary conditions:

$$
B(u, \partial u / \partial r)=0, r \in \Gamma
$$

A is considered a general differential operator, $\mathrm{f}(\mathrm{r})$ is a known analytic function, $\mathrm{B}$ is a boundary operator, and $\Gamma$ is the boundary of the $\Omega$ domain. Operator A can generally be decomposed into linear parts L and nonlinear $\mathrm{N}$. Therefore, Equation (3) can be written as follows:

$$
0(\mathrm{~L}(\mathrm{u})+\mathrm{N}(\mathrm{u}))-\mathrm{f}(\mathrm{r})=0
$$

According to the hematopoietic technique, $v(r, p): \Omega \times[0.1] \rightarrow \mathfrak{R}$ to satisfy the following equations:

$$
H(v, p)=(1-p)\left[L(v)-L\left(u_{0}\right)\right]+p[A(v)-f(r)]=0
$$


That, $p \in[0,1]_{\text {and }} \mathrm{u}_{0}$ an initial conjecture of Equation (2-1) that generally satisfies the boundary conditions.

Obviously from Equation (5):

$$
\begin{aligned}
& \mathrm{H}(\mathrm{v}, 0)=\mathrm{L}(\mathrm{v})-\mathrm{L}(\mathrm{u} 0)=0, \\
& \mathrm{H}(\mathrm{v}, 1)=\mathrm{A}(\mathrm{v})-\mathrm{f}(\mathrm{r})=0,
\end{aligned}
$$

When $\mathrm{p}=0$ equation (6) becomes a linear equation and when $\mathrm{p}=1$, it becomes the original nonlinear equation (1). Therefore, the process of uniformly increasing $\mathrm{p}$ from zero to one is the same process of converting $\mathrm{L}(\mathrm{v})-\mathrm{L}(\mathrm{u} 0)=0$ to $A(v)-f(r)=0$, which is the basis of homotopy. The basic premise of this method is that the answer to Equation (7) can be written as a power series of $\mathrm{p}$ :

$$
v=v_{0}+v_{1} p+v_{2} p^{2}+\ldots
$$

Finally, with the following limit, the approximate answer of Equation (8) can be obtained:

$$
u=\lim _{p \rightarrow 1} v=v_{0}+v_{1}+v_{2}+\ldots
$$

In most cases, it converges to the answer, but the speed of convergence depends on the nonlinear sentence $N(v)$. The second derivative $\mathrm{N}(\mathrm{v})$ must be smaller than $\mathrm{v}$ because the parameter $\mathrm{p}$, when $\mathrm{p} \rightarrow 1$, may be relatively large.

$L^{-1} \partial N / \partial v$ for convergence, the series must be less than 1 .

\section{Statement of the Problem}

The equation expressing this type of vibration is expressed as follows [1]:

$$
\begin{aligned}
& u(0)=A, \dot{u}(0)=0 . \\
& \ddot{u}+\omega^{2} u+\varepsilon u^{2}=0,
\end{aligned}
$$

Where $\omega$ and $u$ are the angular velocities and the displacement of the membrane from the equilibrium position, respectively. A and $\varepsilon$ are also fixed parameters $(\varepsilon \ll 1)$. In this example, the damping force is proportional to the second displacement power. The homotopy function is written as follows:

$$
(1-p)\left[L(v)-L\left(u_{0}\right)\right]+p\left[\ddot{v}+\omega^{2} v+\varepsilon v^{2}\right]=0
$$

Where $L(u)=\ddot{u}+\omega^{2} u$ it is assumed that the initial conjecture of equation (11) is as follows:

$$
u_{0}(t)=A \cos \propto \omega t
$$

Where $\propto(\varepsilon)$ is an unknown constant with $\propto(0)=1$ the sentences of $\mathrm{P}$ coefficient with the same powers after simplification are written as follows:

$$
\begin{aligned}
& L\left(v_{0}\right)-L\left(u_{0}\right)=0, v_{0}(0)=A, \dot{v}_{0}(0)=0 \\
& L\left(v_{1}\right)+L\left(u_{0}\right)+\varepsilon v_{0}^{2}=0, v_{1}(0)=\dot{v}_{1}(0)=0
\end{aligned}
$$


According to the initial conjecture and the approximation of the first order is obtained as follows:

$$
\begin{aligned}
& v_{0}(t)=u_{0}(t)=A \cos \propto \omega t, \\
& \frac{1}{2} \varepsilon A^{2} \cos 2 \alpha \omega t+\frac{1}{2} \varepsilon A^{2}+\ddot{v}_{1}+\omega^{2} A \cos \alpha \omega t+\omega^{2} v_{1}-A \alpha^{2} \omega^{2} \cos \alpha \omega t=0, \\
& \Rightarrow v_{1}(1)=\frac{A\left(4 \omega^{2} \alpha^{2}-A \varepsilon-\omega^{2}+2 \varepsilon A \alpha^{2}\right)}{\left(4 \alpha^{2}-1\right) \omega^{2} \cos \omega t} \\
& +\frac{A\left(\varepsilon A \cos 2 \alpha \omega t-\left(8 \alpha^{2}-2\right) \omega^{2} \cos \alpha \omega t+A\left(\varepsilon-4 \varepsilon \alpha^{2}\right)\right)}{\left(8 \alpha^{2}-2\right) \omega^{2}},
\end{aligned}
$$

In the above answer, sentences with $\cos \omega t$ coefficients are called secular sentences. Secular sentences are sentences that increase indefinitely over time, resulting in divergence. These sentences appear in Stable conservative systems with a periodic solution with the bounded solution. When the excitation frequency is the same as the normal frequency of the system, the resonance phenomenon occurs and this is caused by secular sentences that must be removed from the answer, [11]. Now to determine the constant $\alpha$, set the coefficient $\cos \omega t$ to zero to eliminate secular sentences that may occur in the next iteration.

$$
4 \omega^{2} \alpha^{2}-A \varepsilon-\omega^{2}+2 \varepsilon A \alpha^{2}=0, \Rightarrow \alpha=\frac{1}{\sqrt{2}} \sqrt{1-\frac{\omega^{2}}{\varepsilon A+2 \omega^{2}}} .
$$

As a result, the first-order approximation:

$$
v_{1}(t)=\frac{A}{\left(8 \alpha^{2}-2\right) \omega^{2}}\left(\varepsilon A \cos 2 \alpha \omega t-\left(8 \alpha^{2}-2\right) \omega^{2} \cos \alpha \omega t+A\left(\varepsilon-4 \varepsilon \alpha^{2}\right)\right),
$$

If the first-order approximation is sufficient, the final answer is written as follows:

$$
u(t)=v_{0}(t)+v_{1}(t)=\frac{\varepsilon A^{2}}{\left(8 \alpha^{2}-2\right) \omega^{2}}\left(\cos 2 \alpha \omega t+1-4 \alpha^{2}\right) .
$$

The first-order approximation of the frequency is as follows:

$$
\left.\omega=\sqrt{(1+\varepsilon} A^{2}\right)
$$

\section{Results and Discussion}

In this section of the paper, first, the results obtained from the methods of homotopy and harmonic balance are compared and validated with the reference results [1], the frequency and amplitude changes are examined by changing the effective variables in the problem. Figure (2) shows a comparison of time shifts for the NHBM and HPBM methods with reference [1]. Work presented below is realised by authors from Tehran University and the translation and article edits were carried by Ionut Cristian Scurtu as coauthor of this work. 


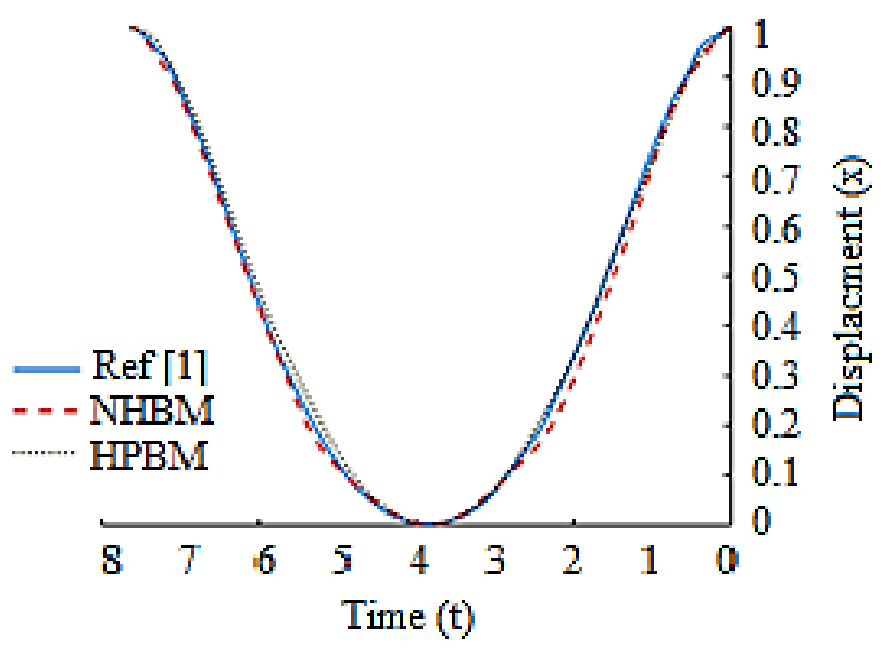

Fig.2. Displacement diagram relative to time.

According to the image, there is a good match between the results of this article and reference [1]. In the following, other parameters are investigated and their effect on the response and frequency of vibrations of the tympanic membrane is investigated. Figure (3) shows the frequency diagram relative to the initial amplitude and Figure (4) shows the three-dimensional graph displacement relative to the initial amplitude and time.
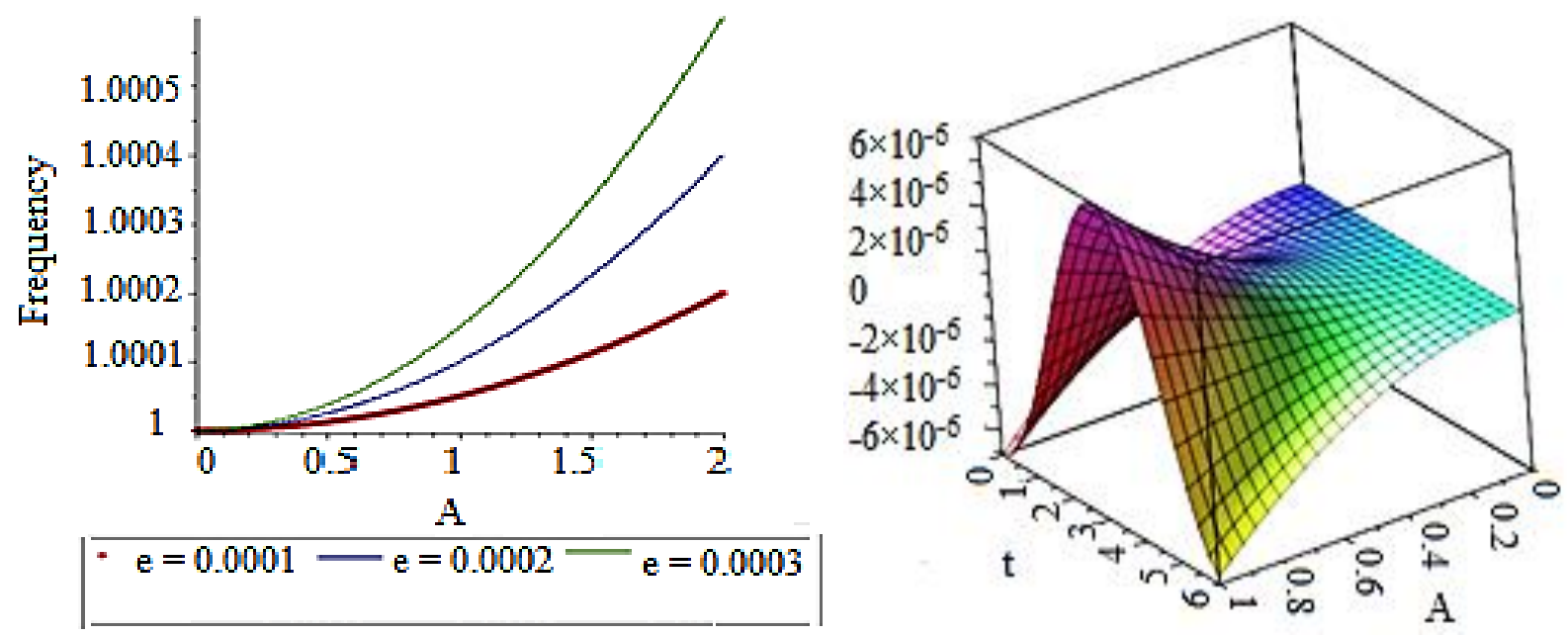

Fig.3. Frequency diagram relative to the initial amplitude.

Fig.4. Three-dimensional displacement graph relative to the time and amplitude.

\section{Conclusion}

The homotopy perturbation method is used to solve the nonlinear equation of vibration of the human tympanic membrane and the homotopy method is easy, simple, and fast and has high accuracy and low computational volume. Comparing the results of this method with the work of other researchers shows that this method has good accuracy. Solving papameters can be updated to different other problems in this domain with valuable results.

\section{References}

[1] Hu, H. Exact solution of a quadratic nonlinear oscillator. Journal of sound and vibration, 450-457, 2006.

[2] Askari, H., Saadatnia, Z., Younesian, D., Yildirim, A., \& Kalami-Yazdi, M. Approximate periodic solutions for the Helmholtz-Duffing equation. Computers \& Mathematics with Applications, 62(10), 3894-3901, 2011.

[3] Wu, B.S., Sun, W.P., Lim, C.W., "An analytical approximate technique for a class of strongly nonlinearoscillators", International Journal of Non-Linear Mechanics, Vol. 41, pp. 766-774, 2006. 
[4] M. M., H. J. M. H. Pashaei., "Solution of strongly nonlinear oscillator problem arising in Plasma Physics with Newton Harmonic Balance Method", Journal of Applied and Computational Mechanics, Vol. 1, No. 2, pp. 59-66, 2015. [5] M. Ghadimi ,H.D. Kaliji., "Application of the Harmonic Balance Method on Nonlinear Equations", World Applied Sciences Journal 22 (4),pp. 532-537, 2013.

[6] Oyedeji, F. O., Erazua, E. A., \& Adeleke, B. B. Gc-mass spectroscopic chemical characterization and physicochemical properties of oil from seed kernels of four cultivars of magnifier indicia. European Journal of Pure and Applied Chemistry Vol, 5(1), 2018.

[7] Preumont, A. Vibration control of active structures: an introduction (Vol. 246). Springer, 2018.

[8] Tang, Y., \& Yang, T. Post-buckling behavior and nonlinear vibration analysis of a fluid-conveying pipe composed of functionally graded material. Composite Structures, 185, 393-400, 2018.

[9] Liao, S. Homotopy analysis method in nonlinear differential equations (pp. 153-165). Beijing: Higher education press, 2012.

[10] Zhang, H., Huang, J., Li, T., Svanberg, S., \& Svanberg, K. Optical detection of middle ear infection using spectroscopic techniques: phantom experiments. Journal of Biomedical Optics, 20(5), 057001, 2015. 